\title{
New Antenna Topologies for 5G Communication Systems
}

\author{
J.M. Fernández González, A. Tamayo Domínguez, M. Sierra Pérez, B. Galocha Iragüen, R. Martinez \\ Rodriguez-Osorio, M. Sierra Castañer.
}

\begin{abstract}
This paper presents new antenna topologies for 5G communication systems. The objective is the study of low losses distribution network technologies and the radiating elements for low profile antennas for 5G applications. Different technologies are considered as PCB technology, LTCC technology and metallized 3D printing in terms of design procedures, transmission losses and manufacturing processes in order to contribute to these types of technologies. Different designs and prototypes are done to validate these antennas for 5G communication systems.
\end{abstract}

Index Terms - Phased Array Antennas, High Frequency Technologies, metalized 3D-printing, substrate integrated waveguide, gap waveguide, LTCC, $5 \mathrm{G}$.

\section{INTRODUCTION}

The arrival of $5 \mathrm{G}$ services demands the use of an increasing bandwidth, which leads to better use of the electromagnetic spectrum. However, the spectrum is widely occupied by other services up to K-band frequencies. Therefore, it is inevitable to use technologies that make the use of spectrum be feasible at Ka-band frequencies and above. $5 \mathrm{G}$ mass deployment requires solutions for working in desired frequency bands with low cost manufacturing. Printed substrate based technologies are good candidates. Nevertheless, printed substrate technologies, as stripline or microstrip, exhibit high transmission losses at high frequencies due to the presence of dielectric. At high frequencies, printed technologies have been replaced by waveguide. This technology is based on electromagnetic waves propagation in a rectangular, cylindrical or elliptical metal cavity, which supposes extremely low transmission losses. However, materials and manufacturing mechanisms are expensive and the resulting structures are heavy and bulky. This makes waveguides incompatible with the development and mass production of small and low cost technology.

In recent years various technologies have been emerged, such as Substrate Integrated Waveguide (SIW) or Gap Waveguide (GW) as Ridge Gap Waveguide (RGW) or Groove Gap Waveguide (GGW), that present the solution through printed structures that emulate the waveguides. These new technologies are becoming globally important because they offer good performance in terms of low weight, low profile and low losses, and a low cost fabrication on a massive scale over other printed technologies or classical waveguides. The SIW technology involves synthesizing a substrate integrated structure with a similar waveguide behavior. To this end, it is used metallized via holes which connect the upper and lower metal plates of the substrate, so that it is obtained a path in which the wave propagates [1-2]. As for the RGW technology, it tries to solve the dielectric losses problems that occur at high frequencies in SIW, due to the wave propagation within a dielectric substrate. RGW uses a waveguide with a central ridge on which the side walls are replaced by a metamaterial surface. This surface prevents the transmission of power in the transverse direction and confines the wave around the central ridge [3-4]. Both technologies have to undergo a process of maturation and consolidation to meet new 5G needs [5-12].

Several Spanish Universities are working in the Project "Enabling 5G", whose general objective is the study of several radio technologies for the future standard of $5 \mathrm{G}$ communications. Three case studies are considered in the project: indoor communication systems, use of satellites in $5 \mathrm{G}$ communications, and vehicle-to-infrastructure communications, at several frequency bands. The main objective of this paper is to propose the design of several antennas for these applications: reflectors with mechanical positioning or active arrays at $\mathrm{Q}$ band for satellite communications, planar antennas at $60 \mathrm{GHz}$ (near field), and MIMO systems at $5 \mathrm{GHz}$ for the vehicle-to-infrastructure communications. Nevertheless, the first step is to analyze the distribution network to compare the transmission losses at high frequencies between different technologies and the radiating elements. Also, to validate the feasibility of these antennas at higher frequencies, a prototype in the $\mathrm{K} / \mathrm{Ka}$ band is designed and will be fabricated.

\section{DISTRIBUTION FEEDING NETWORK}

The main objective of this section is to compare the transmission losses between GGW, RGW, SIW, rectangular waveguide and microstrip technologies at high frequencies. Different models of each technology have been designed and simulated in order to obtain their losses per unity of length in function of the frequency.

In Fig. 1, it is represented the losses for eight types of structures that are: RGW, GGW, SIW filled with dielectric, SIW filled with air, rectangular waveguide filled with dielectric, rectangular waveguide filled with air, microstrip with $1.524 \mathrm{~mm}$ thickness and $0.508 \mathrm{~mm}$ thickness. Microstrip (MS), SIW over dielectric and dielectric-filled waveguide (WG) show similar results due to high losses in the dielectric 
when frequency increases. SIW over air and air-filled waveguide show the best results with losses lower than 2.5 $\mathrm{dB} / \mathrm{m}$ but manufacturing process is more expensive than solutions based on printed technologies. GGW presents transmission losses very close to the losses in SIW and WG filled with air. This can be seen with more detail in Fig. 2. Losses in GGW considerably increases for frequencies from 60 to $100 \mathrm{GHz}$ due to the leakage through the rows of pins are higher. This is because the diameter and separation between pins are constant for all the designed structures for each frequency band.

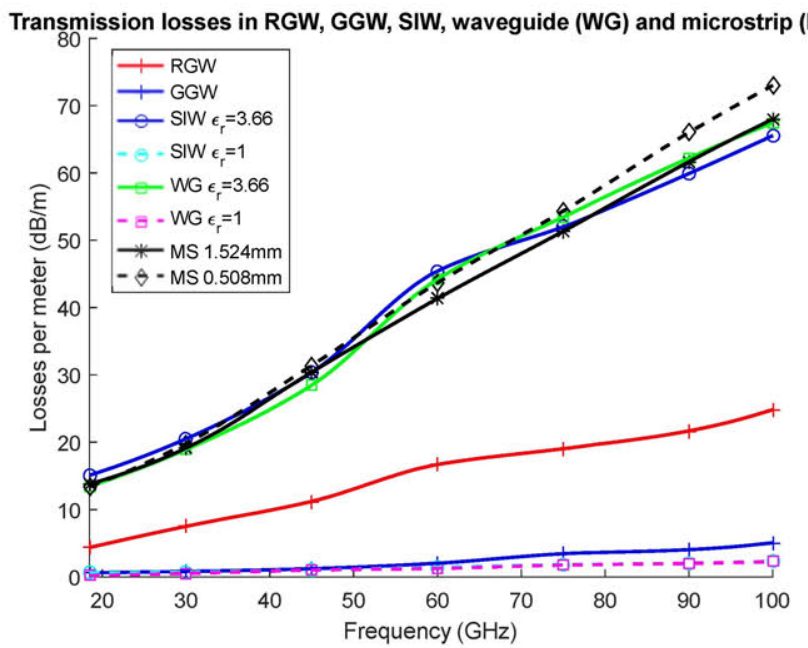

Fig. 1. Transmission losses against frequency.

For higher frequencies, it is necessary to reduce the diameter and the separation to avoid that leakage. RGW presents losses between the dielectric-filled technologies and the GGW and air-filled technologies due to the effect of the ridge conductor and the dielectric at the laterals of the ridge.

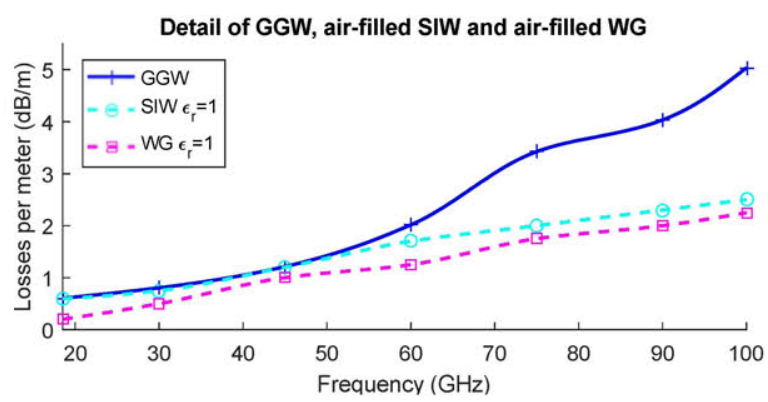

Fig. 2. Detailed transmission losses against frequency.

Losses in RGW and GGW strongly depends on the values selected for the parameters in the structure, especially with the configuration of the rows of pins and, in the case of RGW, the width of the ridge and the thickness of the air gap.

\section{RADIATING ELEMENT}

The option presented in this section is dividing the antenna into passive subarrays, with the minimum possible losses, in order to drastically reduce the number of active integrated circuits like amplifiers or phase shifters [13-23]. Some phase control is needed at the radiating element level and one of the problems is how to obtain information about the losses added in these situations. Here we study the option of several passive subarrays with some control in the main beam direction. Also the circular polarization switch must be implanted at this level.

The proposal shown in fig. 3 is a passive subarray with a variable number of elements from $2 \times 2$ to $8 \times 8$ as a maximum. This range has been selected as a function of the antenna size to obtain the required antenna gain.

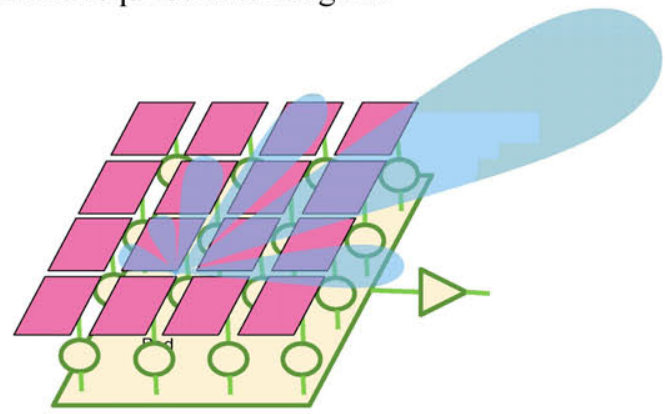

Fig. 3. Subarray schematic circuit.

The circular polarization specified for the antenna includes the option to switch the polarization sense from RHCP to LHCP. To perform this specification a circular printed patch, fed by a metalized via, has been selected as a radiating element. The bandwidth is broadened with a parasitic patch and the coupling to the neighbour elements is minimized with a metallic cavity. Both patches are implemented in a printed circuit over dielectric substrates keeping an air layer in between. The cavity in that air layer is achieved by using a mechanized metallic sheet.

Fig. 4 shows the layers in the antenna element. The feeding is performed through a metalized via to connect the printed strip line to the patch through the ground plane.

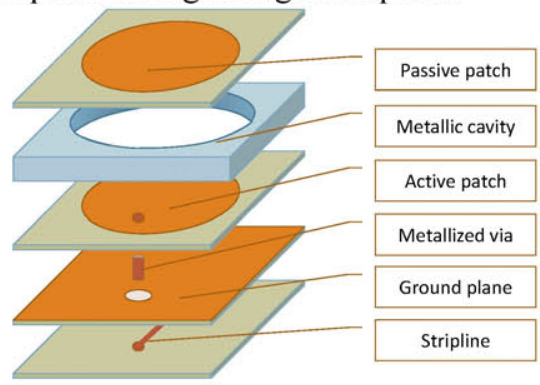

Fig. 4. Printed patch fed with stripline.

To get the appropriate phase at each array element in the main beam pointing process, we need a phase sifter attached at each radiating element. These phase sifters must support 
minimum losses to avoid gain reduction. To reach this goal a two bit phase sifter has been designed. It is based on several switches (PIN diodes, varactors, MEMS ...) to select the feeding point in the printed patch. The subarray feeding structure is especially important to reduce losses as much as possible. Several transmission lines have been studied to perform this structure. Simulations have been done with two dielectric materials: A dielectric substrate like Rogers RO 4003 and a ceramic LTCC substrate like Dupont 9K7V.

\section{CONCLUSION}

High gain phased array antennas at high microwave frequencies or millimeter waves require a large number of elements and a high cost when each element is driven with a low noise amplifier and a phase shifter. To make the antenna viable, passive subarray modules have been designed. The phase control has been limited to two bit phase shifters and the transmission line has been selected to reduce, as much as possible, the distribution losses. The research of inexpensive technologies that provide low transmission losses at frequencies in $\mathrm{Ka}$ band and above is one of the main objectives to address the challenges arising from the development of 5G. In this paper, different technologies have been compared at high frequencies. Prototype construction and measurement results will be presented in IEEE LAMC 2016.

\section{ACKNOWLEDGEMENT}

Simulations done in this work have been performed using CST Microwave Studio Suite 2015 under a cooperation agreement between Computer Simulation Technology (CST) and Technical University of Madrid. The authors want to acknowledge the Spanish Government, Ministry of Economy, National Program of Research, Development and Innovation for the support of the project ENABLING5G "Enabling Innovative Radio Technologies for 5G networks" (Project number TEC2014-55735-C3-1-R) and the project Spacer Debris Radar from Madrid Region Government (Project number S2013/ICE-3000SPADERADAR-CM). Acknowledgement to FPI grant with reference BES-2015075230. We also acknowledge the contribution from the project Jetstream, supported by the call "Retos Colaboración" from the Spain "Ministerio de Economía" (RTC-2015-34957).

\section{REFERENCES}

[1] [1] M. Bozzi, L. Perregrini, K. Wu and P. Arcioni, "Current and future research trends in substrate integrated waveguide technology," Radioengineering, 2009, vol. 18, no 2, p. 201-209.

[2] T. Djerafi and K. Wu, "Substrate integrated waveguide (SIW) techniques: The state-of-the-art developments and future trends," Journal of University of Electronic Science and Technology of China, 2013, vol. 42, no 2, p. 171-192.
[3] E. Alfonso, A. Valero, J.I. Herranz, M. Baquero, M. Ferrando, V. Rodrigo, F. Vico, E. Antonino, M. Cabedo, D. Sánchez, B. Bernardo, A Vila, "New waveguide technology for antennas and circuits," Waves, year 3, ISSN 1889-8297, 2011,pp. 65-75.

[4] E. Alfonso, M. Baquero, P.-S. Kildal, A. Valero-Nogueira, E. RajoIglesias, and J. I. Herranz, "Design of microwave circuits in ridgegap waveguide technology," in Proc. IEEE MTT-S Microw. Symp. Dig., May 2010 , pp. 1544-1547.

[5] F. Xu and K. Wu, "Guided-wave and leakage characteristics of substrate integrated waveguide," Microwave Theory and Techniques, IEEE Transactions on, 2005, vol. 53, no 1, p. 66-73.

[6] J.M. Inclán, M. Sierra. "Planar reconfigurable antennas for satellite communications," ETSI. Telecomunicación. Universidad Politécnica de Madrid, Doctoral Thesis, 2015.

[7] S. Hopfer, "The design of ridged waveguides," IEEE Transactions on Microwave Theory and Techniques, 1955, vol. 3, no 5, p. 20-29.

[8] E. Alfonso, P.-S. Kildal, A. Valero-Nogueira and J. I. Herranz. "Numerical analysis of a metamaterial-based ridge gap waveguide with a bed of nails as parallel-plate mode killer," En Antennas and Propagation, 2009. EuCAP 2009. 3rd European Conference on. IEEE, 2009. p. 23-27

[9] R. E. Collin. "Foundations for microwave engineering," John Wiley \& Sons,p. 117, 2007.

[10] A. Polemi, S. Maci and P.-S. Kildal. "Dispersion characteristics of a metamaterial-based parallel-plate ridge gap waveguide realized by bed of nails," Antennas and Propagation, IEEE Transactions on, 2011, vol. 59, no 3, p. 904-913.

[11] P.-S. Kildal, E. Alfonso, A. Valero-Nogueira, E. Rajo-Iglesias. "Local metamaterial-based waveguides in gaps between parallel metal plates," Antennas and Wireless Propagation Letters, IEEE, 2009, vol. 8 , p. 84-87.

[12] E. Rajo-Iglesias. P.-S. Kildal. "Numerical studies of bandwidth of parallel-plate cut-off realised by a bed of nails, corrugations and mushroom-type electromagnetic bandgap for use in gap waveguides," IET microwaves, antennas \& propagation, 2011, vol. 5, no 3, p. 282-289.

[13] ITU-R SA.364-5 Preferred Frequencies and Bandwidths for manned and unmanned near-earth research satellites.

[14] UIT-R S.580-6 Radiation diagrams for use as design objectives for antennas of earth stations operating with geostationary satellites

[15] ITU-R S.524-9 Maximum permissible levels of off-axis e.i.r.p. density from earth stations in geostationary-satellite orbit networks operating in the fixed-satellite service transmitting in the $6 \mathrm{GHz}, 13 \mathrm{GHz}, 14 \mathrm{GHz}$ and $30 \mathrm{GHz}$ frequency bands.

[16] Manuel Sierra Pérez "Antenas en array activas para comunicaciones y sistemas de localización" XXX Symposium Nacional de URSI. Pamplona 2-4 de septiembre de 2015. España.

[17] R. Baggen, S. Holzwarth, M. Böttcher, B. Sanadgol Phased Array Technology for Mobile User Terminals. Eucap 2011.

[18] S. Vaccaro, D. Llorens del Río, M. C. Viganó Phased arrays with reduced number of phase shifter bits for polarization and pointing control. IEEE APS 2013

[19] Nathan Kundtz, Redmond, Wash. "Next Generation Communications for Next Generation Satellites" Microwave Journal. Augost 2014.

[20] Mikala C. Johnson, Steven L. Brunton, Nathan B. Kundtz, and J. Nathan Kutz "Sidelobe Canceling for Reconfigurable Holographic Metamaterial Antenna" IEEE TAP VOL. 63, NO. 4, APRIL 2015.

[21] Introduction to Reconfigurable Holographic Metamaterial Antenna (RHMA). In Q-Tel Article. 2014

[22] Bahram Sanadgol, Sybille Holzwarth, Alberto Milano, Rafi Popovich "60 GHz Substrate Integrated Waveguide Fed Steerable LTCC Antenna Array" EuCAP 2010

[23] M. C. Viganó, D. Llorens del Río, F. Bongard and S.Vaccaro Sparse Array Antenna for Ku-Band Mobile Terminals Using 1 Bit Phase Controls. IEEE TAP, Vol. 62, No. 4, April 2014A. 